\title{
Agent-based mathematical modeling as a tool for estimating $T$. cruzi vector-host contact rates
}

\author{
Kamuela E. Yong ${ }^{1,2}$, Anuj Mubayi ${ }^{1,3}$, Christopher M. Kribs ${ }^{1,4, *}$
}

June 25, 2015

${ }^{1}$ Simon A. Levin Mathematical, Computational \& Modeling Sciences Center, Arizona State University, Tempe, AZ 85287, USA

2 School of Mathematical \& Statistical Sciences, Arizona State University, Tempe, AZ 85287, USA

${ }^{3}$ School of Mathematical and Natural Sciences, Arizona State University, Phoenix, AZ 85069, USA

${ }^{4}$ Mathematics Department, University of Texas at Arlington, Arlington, TX 76019, USA

* E-mail: kribs@uta.edu

\begin{abstract}
The parasite Trypanosoma cruzi, spread by triatomine vectors, affects over 100 mammalian species throughout the Americas, including humans, in whom it causes Chagas' disease. In the U.S., only a few autochthonous cases have been documented in humans, but prevalence is high in sylvatic hosts (primarily raccoons in the southeast and woodrats in Texas). The sylvatic transmission of $T$. cruzi is spread by the vector species Triatoma sanguisuga and Triatoma gerstaeckeri biting their preferred hosts and thus creating multiple interacting vector-host cycles. The goal of this study is to quantify the rate of contacts between different host and vector species native to Texas using an agent-based model framework. The contact rates, which represent bites, are required to estimate transmission coefficients, which can be applied to models of infection dynamics. In addition to quantitative estimates, results confirm host irritability (in conjunction with host density) and vector starvation thresholds and dispersal as determining factors for vector density as well as host-vector contact rates.
\end{abstract}




\section{$1 \quad 1$ Introduction}

2 At the core of infectious disease transmission are contact processes, through which a pathogen is

3

4

passed from infective to susceptible individuals, whether members of the same population (directly transmitted infections) or host and vector. Implicit in such processes are the movements of individuals, which determine which others are in close enough proximity for the pathogen to be spread. For vector-borne infections in particular, vector dispersal in search of hosts on which to feed is the engine that drives the spread of the disease. For many disease vectors, such as mosquitoes, feeding contacts and dispersal are completely aligned: each bloodmeal typically involves a new host, since hosts are plentiful. Other vectors, however, nest with individual hosts, only leaving in search of a new one when the old host dies or fails to return to the nest. For these vectors, the acts of feeding and dispersal are distinct, and occur with markedly different frequencies, raising the question of which gives the better measure of disease transmission and spread (since repeated contacts between the same host and vector have limited transmission potential). This is the case with the triatomine vectors responsible for the spread of the protozoan parasite Trypanosoma cruzi, the etiological agent of Chagas' disease.

T. cruzi, which is native to the Americas from the southern U.S. down to the southern cone, is carried by numerous species of triatomine vector, most of the genus Triatoma (and each limited to a particular habitat), and transmitted to over 100 mammalian host species. Triatomine bugs pass through five distinct instar stages before reaching adulthood, requiring at least one bloodmeal at each stage. Unlike mosquitoes and many other disease vectors, triatomines' opportunity to feed is limited by host availability and irritability [39, 40]: nymphs typically remain in the nest or den where they hatch, while adults often disperse in search of new hosts [20,32]. Primary hosts such as raccoons (Procyon lotor) and opossums (Didelphis spp.) typically have multiple sleeping places throughout their range and move within that territory to forage, returning to a given den only after 
several days $[35,43]$. (Woodrats move less but have been known to use as many as 5 different nests separated by hundreds of meters [31].) In addition, when too many vectors attempt to feed on a given host, that host takes measures to defend itself, including leaving the den altogether. Since only adult triatomines have wings, and those are more efficient for gliding than flying, it is easier for a host to defend itself against them than against insects such as mosquitoes. Indeed, one modeling study [8] indicated that host irritability plays an important role in vector demographics. When hosts return to a given den or nest often enough, if there are few enough vectors there, they may all be able to feed often enough to survive, mature and reproduce, but when a nest overpopulates, or a host is gone too long, adult triatomines will disperse in search of another host, making use of chemical, thermal, and even infrared cues to detect hosts and infested nests [27]. It is the successful move to a new host, called host switching, that allows T. cruzi to spread, within and even between host populations.

Several modeling studies have already examined the structure underlying host-vector contacts for T. cruzi, in particular how the contact rates saturate in host or vector populations, as a function of the average vector-to-host ratio $[22,23,24,25,26,33]$. (Transmission dynamics are even further complicated by the fact that some hosts, as opportunistic feeders, sometimes prey on the vectors they find nearby, but this study focuses on classical vector-initiated host-vector contacts.) This saturation can affect vector population dynamics, T. cruzi transmission dynamics, and even which strain of $T$. cruzi is advantaged in a cycle where multiple cocirculating strains compete for access to hosts. However, answers to all of these questions require estimates of the basic hostvector contact rates and related quantities, which remain extremely difficult to measure directly in situ. Laboratory studies have investigated individual vector species' preferred feeding frequencies (and average time to defecation, which is key to gauging the efficiency of classical stercorarian transmission of $T$. cruzi) but cannot take into account the real availability of hosts. One notable 
1 study included both lab and field components, measuring the mating duration and frequency, egg 2 size, number and weight, laying and hatching times, hatch proportion and number of ovipositions,

developmental periods and longevity of each stage including adults, feeding preferences on eleven host species including nonmammalian vertebrates, feeding and defecation times, volume and weight of bloodmeals, time to starvation, intraspecific parasitism, T. cruzi infection prevalence, noctural flight activity levels, and host association of three triatomine species (Triatoma sanguisuga, Triatoma gerstaeckeri, Rhodnius prolixus), in some cases as a function of temperature [34]. However, it did not capture host-vector contact frequencies in the field. (It also revealed some discrepancies between laboratory and field-based measures of the same quantities.) A recent study provided a comprehensive review of host and vector demographics and T. cruzi prevalence in species present in the U.S. [25], but the average host-vector contact rates could only be back-estimated assuming observed prevalences represented endemic steady states, and even then the rates incorporated probability of infection given a contact. In cycles where multiple contact-related transmission avenues exist (e.g., stercorarian and oral), direct estimates of these rates remain key to understanding $T$. cruzi transmission.

Given the difficulties inherent in direct measurement, mathematical models offer one potential way to estimate contact rates using the basic behavioral tendencies measured in laboratory studies. Compartmental models have long been used to describe the collective behaviors of populations using relatively little biological information; however, in recent years agent-based modeling has developed as a means of using more detailed information at the individual level to build up emergent properties of systems comprising many individuals [1, 18]. As Gaff wrote [14], "Agent-based models, also called individual-based models, are computer-based models that simulate the actions and interactions of autonomous agents that represent the individuals of the population." The activity of these agents can be compiled to obtain population-level measures of a biological system at a given scale. Gaff, for 
1 instance, developed an agent-based model (ABM) of deer-tick interaction [14] in which tick vectors 2 (Ixodes spp.) passed through four life stages (egg, larva, nymph, adult), with each transition 3 requiring a single bloodmeal, and found that the ABM predicted significantly lower infection rates 4 than a classical ordinary differential equations (ODE) model with analogous parameter values, due 5 to the ABM's discrete, limited host interactions for each vector (contrasted with the continuous 6 host-vector interactions implied by the ODE system). ABMs have also been used to study T. cruzi 7 transmission to humans (Chagas disease). A group of French researchers $[2,44]$ used a spatially

explicit cellular automaton model with discrete individuals to study spatial infestation patterns of a Mexican rural village by the T. cruzi vector Triatoma dimidiata, finding that the constant influx from the forest surrounding the village made the village's periphery the most important place to apply vector control (insecticide). Galvão et al. used a within-host ABM to study the impact of $T$. cruzi at the cellular level on cardiac tissue regeneration following a bone marrow stem cell transplant. Devillers et al. [13] used an ABM to study the competition for hosts between two T. cruzi types in South America, finding that sylvatic reservoir hosts were necessary in order to explain the observed prevalences in humans. The present study thus proposes to use known information about the behaviors of individual sylvatic hosts and vectors to study the basic contact mechanisms (vector feeding and host switching) responsible for the spread of T. cruzi in sylvatic reservoir cycles, through the framework of an ABM. Model simulations will use the contexts of raccoons and (separately) woodrats (Neotoma micropus), common hosts in the southern U.S., and their associated vectors T. sanguisuga and T. gerstaeckeri [21], although the model structure can be applied to other hosts and vector species as well.

The primary research questions are thus: How often do vector-host contacts really occur? and what impact does host irritability have on that rate? (A related question is, how often does host switching occur within a given cycle?) Underlying these questions is the more complex issue of 
1 how best to use these baseline rates to describe the rate at which T. cruzi actually spreads. The following section details the model structure, built around the key elements of host and adult

\section{Model}

The ABM developed for this study was coded in NetLogo 5.0.3 [46]. The model description in this section follows the ODD (Overview, Design concepts, Details) protocol for describing ABMs $[17,19]$ using the complete required format.

\subsection{Purpose}

The ABM developed for this study uses information from the scientific literature about the demographics and movement of individual hosts (raccoons or woodrats) and vectors (T. sanguisuga and/or T. gerstaeckeri), and about the feeding preferences of vectors, to estimate the rates at which vectors feed on hosts and switch hosts in the field, as a function of host irritability level (maximum number of bites tolerated by a host in one night) and transmission cycle (host species). The resulting estimates will inform the current understanding of how the parasite Trypanosoma cruzi spreads at the population level in sylvatic settings.

\subsection{Entities, state variables, and scales}

The entities (agents) in the model include hosts (raccoons and woodrats), vectors (T. sanguisuga and T. gerstaeckeri), and homes (raccoon dens and woodrat nests). Hosts have counters for the number of vectors of each species trying to bite them on that time step (for purposes of determining 
1 host irritability-the counters are reset at each time step). Vectors likewise have counters marking 2 whether or not they try to bite a given host type during the present time step; hunger counters,

marking the number of turns (days) since last feeding; variables recording the types of patch in which they currently and most recently resided, for purposes of marking host type switching across cycles (not implemented in this study); and two lists describing their personal feeding histories, marking the time-index and host-ID at each feeding (stored to a global list at death). Homes have counts of the number of juvenile vectors of each species living there, as well as a host absence counter, which also serves as a hunger counter for the juveniles. For simplicity, homes are assumed permanent, created at setup and never destroyed. All agents also have $(x, y)$ coordinates (for homes these are fixed).

The world consists of a $75 \times 75$ grid, with opposite boundaries identified for continuity (left to right and top to bottom, as is commonly done, but note this creates a torus geometry). Cell diameters were set at $10.4 \mathrm{~m}$, since this reflects the approximate average minimum distance between woodrat nests as well as mean distance traveled daily by a woodrat [38, 37, 45]; thus the model simulates an area of about 60 ha or 150 acres. Each patch (grid cell) has a landscape property taking on one of three possible values (permanent over the course of a simulation): wooded (raccoon habitat), prickly pear cactus (woodrat habitat), or neither. For the purpose of this study, simulations were run with all cells of the same landscape type (wooded for raccoons, or prickly pear for woodrats).

The time step used is one day, since host-vector contacts only occur when hosts return to their sleeping places to rest after foraging. Each simulation was run for 100 days. 


\section{$1 \quad 2.3 \quad$ Process overview and scheduling}

2 Following setup (q.v.), each day the following five routines occur in the order given below, until 3 either the specified number of days passes or there are no more vectors, whichever occurs first:

- hosts (woodrats and raccoons) move;

- vectors of each type move (disperse if hungry enough);

- host births and deaths;

- vector natural death, feeding, and starvation;

- homes (nests and dens) are updated: primarily juvenile vector birth, death and maturation.

\subsection{Design concepts}

Basic principles: In accordance with research literature, raccoons are assumed to be solitary and have several sleeping places across their territory, while woodrats have relatively few and may share a nest (generally of the other gender, as for breeding, but in this model gender is not made explicit, and nests may simply hold up to two woodrats at a time). Juvenile vectors of either species are assumed incapable of dispersal (since they have no wings and are commonly found in nests or dens), while adult vectors are assumed to disperse only to avoid starvation. Hosts are assumed to be able to defend themselves and/or leave a home in order to receive no more than a given number of bites per night (the host irritability threshold). Bites by juvenile vectors are assumed to provoke less irritation than bites by adult vectors, by a given factor.

Emergence: The model's primary outputs are measures of vector feeding and host-switching rates, at the population level but compiled from individuals.

Adaptation: In foraging from day to day, hosts are assumed to seek (and find) the least-recently visited site within a day's range; hosts are also assumed able to defend themselves against excessive 
1 bites per night. Adult vectors are assumed to get hungry a certain number of days after feeding 2 (threshold 1), disperse in search of a (new) host after another period (threshold 2), and finally

starve if they have not fed after another period (threshold 3); when dispersing, adult vectors are also assumed able to find occupied homes within a day's travel radius.

Sensing: Hosts are assumed to be able to detect which part(s) of their home ranges (represented by dens or nests) within a single day's travel have had longest to replenish food sources. Vectors are assumed to be able to detect their own hunger thresholds, and dispersing vectors are assumed to be able to detect hosts located within a single day's travel of their present locations.

Interaction: The primary interactions of interest involve vectors feeding on hosts, within the confines of a den or nest, subject to the host irritability threshold (thereby making competition for access to hosts an indirect interaction between vectors). Births of new hosts or juvenile vectors require the presence of an adult of the given species, an indirect interaction.

Stochasticity: In setup (q.v.), the spatial configuration of landscape types (but not the numbers of cells of each landscape type) is random, as are which specific nests and/or dens are infested with vectors (but not the numbers of each that are infested), which nests/dens serve as initial locations for the hosts, and which infested nests/dens serve as initial locations for the adult vectors. The initial value for each home's host absence counter is taken from a uniform distribution bounded above by the vector dispersal threshold. Initial numbers of juvenile vectors of each species in infested homes are drawn from exponential distributions with the corresponding expected means.

During runtime, juvenile vector births, survival, and maturation, adult vector survival, and host birth and survival are all taken from binomial distributions; once the numbers are determined, which host or adult vector dies is random. Newborn hosts are placed randomly in a home of the appropriate type with room for them. Host movement (from one home to another), when not triggered by host irritability (excessive bites), is determined stochastically with a given probability, 
and when either (a) there are multiple homes within a day's travel for that host with maximal host absence counter, or (b) there are no unoccupied homes within a day's travel but there are homes with room for the given host, the home to which the host moves is chosen randomly. When an adult vector disperses without reaching an occupied home immediately, its distance traveled in a day is drawn from an exponential distribution, and its direction is random. When too many vectors attempt to bite a given host, which adult vectors are denied feeding is chosen randomly.

Observation: At the end of each simulation, the collected individual feeding histories (timestamp and host ID for each successful feeding, as well as timestamps of birth and death, from which one can determine feeding and host-switching rates) of all adult vectors are stored for analysis, as well as the collected dispersal results. In addition, NetLogo's Behavior Space allows one to tabulate the number(s) of agents in various states at each time step. In this study the numbers of vector bites, hosts, and vectors were recorded at each time step in order to calculate total instantaneous per-host or per-vector contact rates.

\subsection{Initialization}

Setup begins by assigning each grid cell a landscape type (wooded raccoon habitat, prickly pear cactus woodrat habitat, or neither): each of the two habitat types has a preset percentage of cells, and the corresponding number of cells is then chosen randomly from the grid and assigned. Next appropriate numbers of woodrat nests and raccoon dens are created, based in each case on the number of homes per host and number of hosts, of the given type. Each home is randomly placed on a grid cell of the appropriate habitat type not already occupied by a home. Since homes will later be seeded with vectors, each home's host absence counter is assigned by a draw from a uniform distribution on $\left[0, t_{h}\right]$, where $t_{h}$ is the vector dispersal hunger threshold (if the host absence counter exceeded $t_{h}$, the adult vectors would all have left). 
Next hosts are created (raccoons and woodrats), each according to the preset densities (and habitat size), and placed randomly in unoccupied homes (woodrats are allowed to be placed up to 2 in a nest, as with breeding pairs although no genders are assigned explicitly). Their bite counters are initialized to 0 .

Homes are then infested with juvenile vectors according to preset infestation percentages for each home type. The number of juvenile T. sanguisuga in an infested nest is drawn from an exponential distribution whose mean is calculated as follows: The given per-area vector density is multiplied by the area of the total grid (in $\mathrm{m}^{2}$ ), scaled down by the [preset, initial] proportion of vectors which are juveniles, by the proportion of the grid which consists of woodrat habitat, and by the [preset, initial] proportion of vectors in a nest which are T. sanguisuga. The numbers of juvenile T. gerstaeckeri in an infested nest, and of juvenile T. sanguisuga in an infested den, are calculated similarly. Analogous (but simpler) calculations yield the [deterministic] numbers of adult vectors of each species in the entire grid; the corresponding adult vectors are then created and randomly placed in homes already infested with juveniles of the given species, once their counters and lists are initialized (to 0). Their patch types are also then set to mark their initial host species affiliation.

Finally, the global result lists that will store dispersal outcomes and vector feeding histories are initialized (as empty).

\subsection{Input data}

The model does not use input data to represent time-varying processes. However, in order to explore the effect of the host irritability threshold on vector feeding rates, host irritability was varied across experiments (each experiment consisting of 100 simulations using identical parameter values), set at either 2 or 10 bites per night. In addition, one set of experiments was run with raccoons as the only hosts and T. sanguisuga as the only vectors, while a different set of experiments involved 
1 woodrat hosts only, and both species of vector (since both are found in woodrat nests).

2 Model parameters, available as user inputs in interactive mode, are summarized in Table 1 and

3 addressed in the following subsection.

\section{${ }_{4} \quad 2.7 \quad$ Submodels}

5

1. Move hosts

(a) Move woodrats

Each woodrat is polled in turn to determine whether the total number of attempted vector bites (calculated as the sum of three quantities: the number of adult T. sanguisuga in the present nest which wish to feed as evidenced by their woodrat bite counters [being positive], the number of adult T. gerstaeckeri in the nest which wish to feed, and the total number of juvenile vectors in the nest, rescaled both for relative irritation and desired feeding frequency) meets or exceeds the host irritability threshold. If not, then a random draw (from a uniform distribution on the unit interval) is compared with the set probability of switching nests (if less, host dispersal should occur regardless of irritability). When either of the two trigger conditions is met, if any unoccupied nests are within a day's travel, the woodrat moves to a randomly chosen one from among those longest-unoccupied (i.e., with greatest host absence counter). Otherwise, when dispersing, the woodrat moves to any randomly chosen nest with no more than 1 woodrat presently occupying it.

(b) Move raccoons

Raccoons decide whether, and where, to move according to the same algorithm as for woodrats, except that dens have no T. gerstaeckeri, and raccoons have different travel distances and probabilities than woodrats. 


\begin{tabular}{|c|c|c|c|}
\hline Parameter & Definition & Value & Reference \\
\hline grid size & length \& width of modelled area & 75 cells & $\mathrm{N} / \mathrm{A}$ \\
\hline cell_diameter & length \& width of a grid cell & $10.4 \mathrm{~m}$ & {$[37,38,45]$} \\
\hline percent_wooded & proportion of cells which are raccoon habitat & 0 or 100 & $\mathrm{~N} / \mathrm{A}$ \\
\hline percent_prickly & proportion of cells which are woodrat habitat & 100 or 0 & $\mathrm{~N} / \mathrm{A}$ \\
\hline raccoon_dns & initial density of raccoons & $0.08 \frac{r a c}{a c r e}$ & {$[25]$} \\
\hline woodrat_dns & initial density of woodrats & $9.3 \frac{\text { acre }}{\text { acre }}$ & {$[25,37]$} \\
\hline dens/raccoon & ratio of dens per raccoon & 9.25 & [43], App. \\
\hline nests/woodrat & ratio of nests per woodrat & 2.1 & {$[31]$} \\
\hline den_infest_prop & $\%$ of dens initially infested with vectors & $70.5 \%$ & App. \\
\hline nest_infest_prop & $\%$ of nests initially infested with vectors & $70.5 \%$ & {$[12,34]$} \\
\hline vec_density & vector population density & $128 \frac{v e c}{a c r e}$ & {$[6]$} \\
\hline juvprop & [initial] proportion of vector pop. which are juveniles & 0.4785 & {$[12,34]$, App. } \\
\hline nestGprop & $\begin{array}{l}\text { [initial] proportion of vectors in a woodrat nest } \\
\text { which are T. gerstaeckeri }\end{array}$ & 0.4 & {$[12,34]$, App. } \\
\hline$t_{b}$ & $\begin{array}{l}\text { maximum number of bites a host can tolerate } \\
\text { before leaving due to irritation }\end{array}$ & $\begin{array}{l}2-10 \frac{\text { bites }}{\text { night }} \\
\text { (varies) }\end{array}$ & [8] \\
\hline juv_intense & \# of juvenile vector bites equiv. to an adult bite & 2.06 & {$[8,34]$, App. } \\
\hline$m_{r}$ & daily probability a raccoon changes den & 0.685 & {$[38,43]$} \\
\hline$m_{w}$ & daily probability a woodrat changes nest & 0.25 & App. \\
\hline$d_{r}$ & maximum distance a raccoon travels in one day & $990 \mathrm{~m}$ & {$[38,43]$} \\
\hline$d_{w}$ & maximum distance a woodrat travels in one day & $27.14 \mathrm{~m}$ & {$[37,38]$} \\
\hline$t_{w}$ & vector bloodmeal digestion time (min. time btw. bites) & 7 days & [3], App. \\
\hline$t_{h}$ & $\begin{array}{l}\text { time since last feeding before a vector disperses } \\
\text { to seek a new host }\end{array}$ & 14 days & {$[15,34]$} \\
\hline$t_{s}$ & $\begin{array}{l}\text { maximum adult vector survival time without feeding } \\
\text { (starvation threshold) }\end{array}$ & 36 days & [34], App. \\
\hline mean_vec_travel & average daily dispersal distance for adult vectors & $1000 \mathrm{~m}$ & App. \\
\hline$R a c \operatorname{con} C C$ & density carrying capacity for raccoons & $0.144 \frac{\text { rac }}{a c r e}$ & [25] \\
\hline Woodrat $C C$ & density carrying capacity for woodrats & $21 \frac{w r}{a c r e}$ & {$[25]$} \\
\hline$p_{b}^{r}$ & daily prob. that a raccoon births one child & 0.00246 & [25], App. \\
\hline$p_{b}^{w}$ & daily prob. that a woodrat births one child & 0.00492 & [25], App. \\
\hline$p_{s}^{r}$ & daily raccoon survival probability & 0.99891 & [25], App. \\
\hline$p_{s}^{w}$ & daily woodrat survival probability & 0.99727 & [25], App. \\
\hline$p_{b}^{s}$ & daily prob. that an adult $T$. sanguisuga hatches a juv. & 0.137 & [25], App. \\
\hline$p_{b}^{g}$ & daily prob. that an adult T. gerstaeckeri hatches a juv. & 0.355 & [25], App. \\
\hline$p_{s j}^{s}$ & daily survival probability for T. sanguisuga juveniles & 0.99818 & [25], App. \\
\hline$p_{s j}^{g}$ & daily survival probability for $T$. gerstaeckeri juveniles & 0.99586 & {$[25,30]$, App. } \\
\hline juv_sta & maximum juvenile vector survival time $\mathrm{w} / \mathrm{o}$ feeding & 31.5 days & [34], App. \\
\hline$p_{d}^{s}$ & daily maturation prob. for T. sanguisuga juveniles & 0.00122 & {$[25,34]$, App. } \\
\hline$p_{d}^{g}$ & daily maturation prob. for $T$. gerstaeckeri juveniles & 0.00276 & [34], App. \\
\hline$p_{s a}^{s}$ & daily survival probability for $T$. sanguisuga adults & 0.99999 & [25], App. \\
\hline$p_{s a}^{g}$ & daily survival probability for $T$. gerstaeckeri adults & 0.99650 & [34], App. \\
\hline
\end{tabular}

Table 1: User input parameters (App. refers to Appendix) 


\section{Move vectors}

(a) Move T. gerstaeckeri

Each adult vector, in turn, disperses if its hunger counter meets or exceeds the dispersal hunger threshold. If any occupied nests are within a day's travel (distance drawn from an exponential distribution with the given mean) the vector enters the nearest one. In this case, the vector's previous and current patch types, and the global list of dispersal outcomes, are updated. If no occupied nests are within reach, the vector travels a random distance (from the exponential distribution) in a random direction.

(b) Move T. sanguisuga

T. sanguisuga disperse like T. gerstaeckeri except that they are assumed to prefer raccoons over woodrats, so when dispersing, if there is an occupied den within a day's travel the vector enters the nearest one; if not, then the vector checks for occupied nests as before.

3. Update hosts

(a) Update woodrats

First the number of woodrats to be born is drawn from a binomial distribution where $n$ is the number $N$ of woodrats on the grid and $p$ is the baseline birth probability scaled by the logistic factor $(1-N / K)$ (where $K$ is the carrying capacity). The given number of newborn woodrats is created, each randomly placed in a nest with room for it, and its counters initialized to 0 .

Next the number of woodrats to survive is likewise drawn from a binomial distribution with a given probability. The number to die is then calculated by subtraction, and the given number of woodrats are chosen randomly to die. 
(b) Update raccoons

Raccoon births and deaths are determined similarly to woodrats, but with their own probabilities.

4. Update vectors

(a) Update T. gerstaeckeri (TG)

First, the number of natural deaths of adult TG vectors on the grid is determined (by subtracting from the total number a random draw from a binomial distribution with given survival probability). For the given number of deaths, the vectors are selected randomly, their feeding histories are ended and appended to the global lists of feeding histories (host IDs and times), and they die.

Next, the surviving vectors' and woodrats' woodrat-TG bite "counters" are reset to 0, and each vector is examined concurrently to determine if (a) its hunger counter meets or exceeds the feeding hunger threshold and (b) there is a woodrat there. If so, its bite counter, and the corresponding one of a (randomly chosen if not unique) woodrat there are incremented by 1 . Once all adult TG have been polled, any woodrats with positive TG bite counters are polled concurrently to determine host irritability. For those woodrats, the number of adult T. sanguisuga in that nest with hunger counter meeting or exceeding the feeding hunger threshold is added to the total number of juvenile vectors in the nest rescaled for relative intensity and frequency of bite; if the sum exceeds the host irritability threshold, then the host is considered irritated, and the number of successful adult TG feeding is set at the host irritability threshold multiplied by the proportion of desired bites which were adult TG (the product is rounded down to a whole number). The number of unsuccessful TG biters (calculated by subtraction) is then determined, 
and that many attempted biters then have their bite counters reset back to zero.

Finally, the adult vectors are again polled concurrently: the successful biters have their hunger counters reset and their feeding histories updated, and those who did not feed have their hunger counters incremented, and those whose hunger counters meet the starvation threshold have their feeding histories retired and appended to the global lists, and then they die.

(b) Update T. sanguisuga (TS)

First, natural death of adult vectors is determined, as for TG but with a different survival probability. Next, host and vector TS bite counters are reset. Then the vectors are polled concurrently to see if their hunger counters meet or exceed the feeding threshold. If a woodrat is present there, the vector tries to bite it (host and vector bite counters are incremented); if a raccoon is present there, the vector tries to bite it (order is irrelevant since woodrats and raccoons do not share habitat). Following this, woodrats are polled concurrently to see which have positive TS bite counters. For those that do, their host irritability is determined, by comparing to the irritability threshold the number of attempted bites (calculated as the sum of the woodrat's two species-specific vector bite counters plus the total number of juvenile vectors there, rescaled as before). For irritated hosts, the number of successful TS biters is calculated as the irritability threshold multiplied by the proportion of attempted bites made by adult TS. The appropriate number of unsuccessful biters are chosen at random and their bite counters reset. Then raccoons are similarly checked for irritability (though they have no attempted TG bites to tally). Finally, each adult TS is polled concurrently either to reset or to increment their hunger counter, and those who did not feed and now have a hunger counter at the starvation threshold die as detailed for TG. 
(a) Update nests (juvenile vectors)

Woodrat nests are polled concurrently: if unoccupied, their host absence counters are incremented, and if the host absence counter meets the juvenile starvation threshold, the juveniles in the nest die. If occupied, the host absence counter is reset to zero. Next, the number of juvenile vectors of each species in that nest is incremented by a draw from a binomial distribution with $n$ the number of adult vectors of the given species in the nest, and $p$ the species' birth rate. Next, the number of juvenile vectors of each species in the nest surviving to the next time step is determined by a draw from a binomial distribution with given probability. Then the number of juvenile vectors of each species there maturing into adults is also determined by a draw from a binomial distribution with given probability; the corresponding adult vectors are created, with properties appropriately initialized, and the number of juveniles there of that type is decremented correspondingly.

(b) Update dens (juvenile vectors)

The host absence counter and juvenile vector population of each raccoon den are updated concurrently in a similar way as for nests, but with different probabilities and no $T$. gerstaeckeri.

Parameter estimates given in Table 1 are discussed in more detail in the appendix, and in a separate literature review [38]. 


\section{Results}

2 Initial analysis was made from 20 simulations for each of the four scenarios (raccoon or woodrat

cycles; host irritability threshold $t_{b}=2$ or 10 bites per host per night), and then extended to 100 simulations. In all simulations the host populations held steady near their initial [equilibrium] values (and will therefore not be further discussed), but the vector densities varied substantially over time. Juvenile biting rates were computed (and are here reported) in units of adult-equivalent bites, and should be multiplied by the equivalence factor of 2.06 given in Table 1 to get the number of juveniles per host or vector per night that fed.

Raccoons: The relatively low host density (compared to woodrats) coupled with a low host irritability threshold severely curtails vectors' ability to feed, with mass starvation dropping adult vector density from 10,000 over the entire grid to a steady mean (across simulations) of just 662.5 $\left(t_{b}=2\right)$ or $1630\left(t_{b}=10\right)$. The juvenile TS population, which starts out in growth mode, undergoes a similar reset, dropping an order of magnitude from a high of nearly 20,000 to a final mean of $2023\left(t_{b}=2\right)$ and $3900\left(t_{b}=10\right)$. The resulting per capita biting rates for juvenile TS fall during the initial growth period but recover when the adult population undergoes starvation, thereafter leveling out at a fairly steady $0.000978\left(t_{b}=2\right)$ and $0.003654\left(t_{b}=10\right)$ adult bites per juvenile per day. The per capita adult TS biting rates, on the other hand, begin low and then rise when the juvenile population begins to starve as well, settling at a markedly higher 0.03735 and 0.04600 adult bites per adult per day. From the host's perspective, meanwhile, we see three phases: if $t_{b}=2$, the average host receives $1.5-1.75$ bites per night (less than the maximum of 2 since raccoons spend some nights in uninfested dens), first split 40/60 juveniles/adults, then mostly juveniles, and finally nearly all adults. Graphs for all of these trends are given as an example in Figure 1 (for $t_{b}=2$; the overall shape is similar for $t_{b}=10$ ). The fact that the total number of bites an average host received per night for $t_{b}=10$ remained well above 9 throughout the reset period underlines the key 

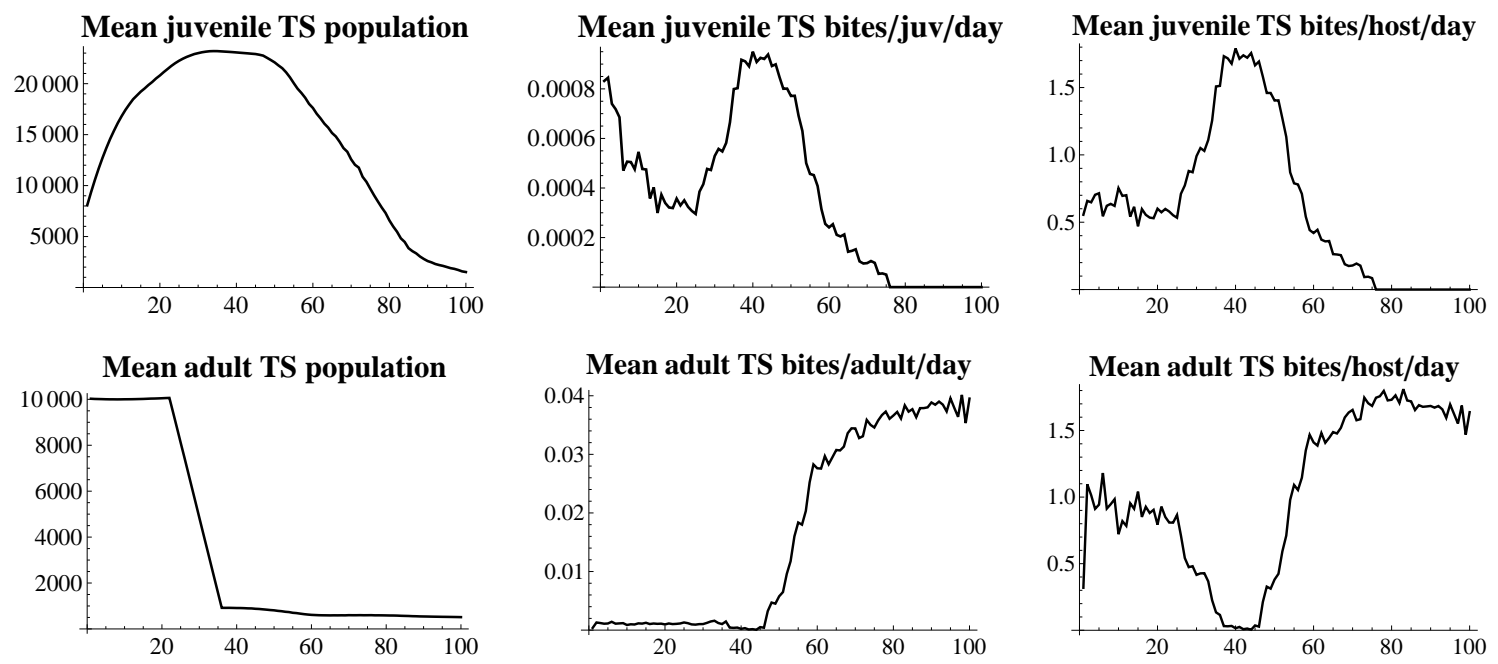

Figure 1: Graphs of mean (top) juvenile and (bottom) adult TS densities and biting rates over time, for 100 days, on raccoons with $t_{b}=2$ bites/raccoon/day: left, vector densities; center, per-vector biting rates; right, per-host biting rates (rates in adult bites per day)

role played by host irritability in controlling vector density, and the average interval between bites for a given vector, which may be calculated as the reciprocal of the corresponding per capita rate, offers a key measure of survivability when compared with the starvation threshold (from Table 1, 36 days for adults and 31.5 days for juveniles): The interval remains well above 100 days for juveniles over time for both $t_{b}=2$ and $t_{b}=10$, indicating that the relative scarcity of raccoons and the consequent infrequency of their visits to each den are not enough to sustain substantial juvenile populations (caveat: this model does not take into account the visits to these same dens made by other hosts such as opossums and skunks), while for adult vectors the mean feeding interval drops from over 120 days to under 27 days for both $t_{b}$ values, indicating arrival at a sustainable level. In summary, under such difficult feeding conditions vector densities inevitably drop, with adult bites dominating juvenile feeding at the final levels.

Woodrats: The relative abundance of woodrats meant in simulations that regardless of irritability threshold they visited infested nests often enough to prevent the mass juvenile starvation that 
occurred in raccoon dens, with the result that the juvenile populations of both TS and TG showed steady growth (although it was concave down for $t_{b}=2$ and concave up for $t_{b}=10$ ). It should be noted that this is in part a limitation of the model, which for computational efficiency calculated [successful] juvenile bites but determined juvenile starvation on a per-nest, not per-juvenile, basis. Under low irritability threshold $\left(t_{b}=2\right)$, the adult vector densities for both species still underwent a starvation reset between 23 and 37 days; the TS mean dropped from 6053 to a more or less level 1540 vectors across the grid, while the TG density quickly returned to growth mode which continued past 100 days. With hosts both plentiful and more bite-tolerant $\left(t_{b}=10\right)$, the simulations showed consistent and accelerating growth for juvenile and adult vector densities of both species, suggesting that the true value of $t_{b}$ is lower. Per capita biting rates for $t_{b}=2$ for both juvenile and adult TS declined steadily over time, with respective means of 0.02680 and 0.009870 bites per day per vector over the 100 days, both too low to beat starvation, suggesting a struggle - at least in the short term - for TS under these conditions. The TG rates leveled off for both juveniles and adults at $t_{b}=2$, but at even lower levels, 0.006354 and 0.00207 bites per vector per day, suggesting that most vectors do starve, compensating for the high reproduction rate. Mean per-host juvenile biting rates were 0.5333 (TS) and 0.3003 (TG) per night over 100 days, and around 0.01 for adults. The per capita biting rates for a high biting threshold $\left(t_{b}=10\right)$, meanwhile, declined over time due to competition with growing numbers, but at rates of 0.06445 and 0.0678 bites per vector per night for TS (resp. juveniles and adults) and 0.04261 and 0.04873 for TG, all well above the critical rate to avoid starvation. Although the juvenile bites dominate the adult bites in absolute number due to their higher densities, the totals per host per night still fall well short of the bite ceiling, suggesting that if woodrats were able to tolerate so many bites per night their associated vector densities would be markedly higher. For comparison purposes, the mean adult vector densities for both species and both irritability thresholds are graphed in Figure 2 (note vertical scales differ). 
Mean adult TS population, $\mathrm{tb}=2$

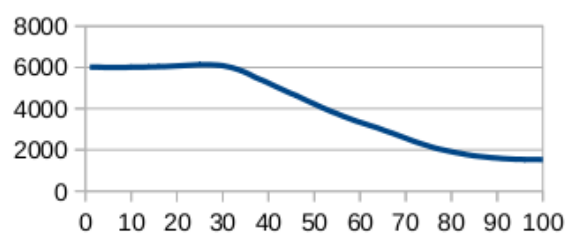

Mean adult TS population, $\mathrm{tb}=10$

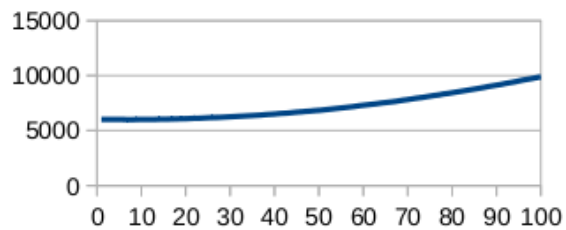

Mean adult TG population, $\mathrm{tb}=2$

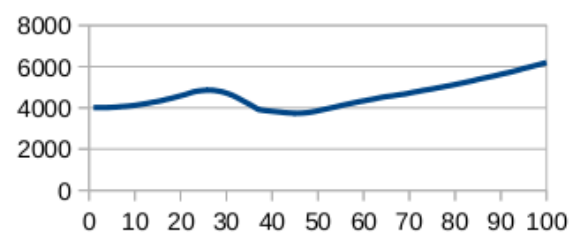

Mean adult TG population, $\mathrm{tb}=10$

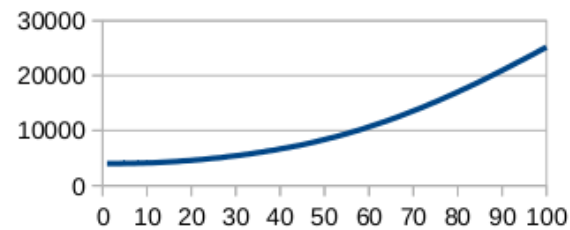

Figure 2: Graphs of mean adult vector densities over time for 100 days associated with woodrats with (top) $t_{b}=2$, (bottom) $t_{b}=10$ : left, TS; right, TG

1

Lastly, we consider the outcomes of the adult vector dispersals motivated by hunger. Figure 3 presents the six histograms showing the frequency of duration of dispersals from 1 to 23 days (with 23 days, the last bar, representing starvation); underneath each histogram is given the proportion of failed dispersals and mean duration of successful dispersals. As can be seen in the figure, although the increase in host tolerance reduces both the proportion of failure and the mean length of successful dispersal, it has a much more dramatic effect on vectors associated with woodrats than on those associated with raccoons. In both vector species, starvation prior to finding a new host becomes extremely rare, and it takes on average much less time to find one. This is consistent with the significant difference seen in vector densities and biting rates as woodrat tolerance increases, relative to the impact of $t_{b}$ on raccoon-associated vectors.

A preliminary look at the results of the simulations performed for this study suggests that in practice dispersal in search of hosts is not only common (consistent with observations in the literature, e.g., [34]) but usually does result in switching to a new host (rather than finding the previous one in a new location). Although this model ignores territoriality in hosts, which may 


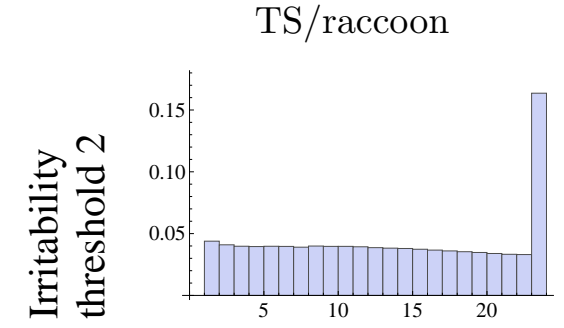

\%starve: $16.36 \%$

MDSD: 11.08 days

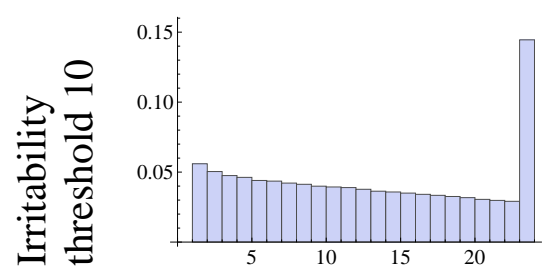

\%starve: $14.45 \%$

MDSD: 10.41 days
TS/woodrat

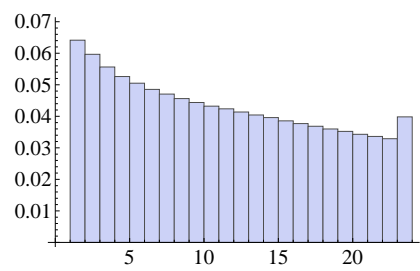

\%starve: $3.98 \%$

MDSD: 10.32 days

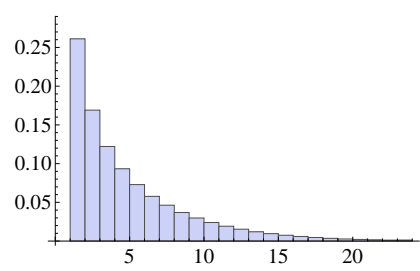

\%starve: $0.13 \%$

MDSD: 4.374 days
TG/woodrat

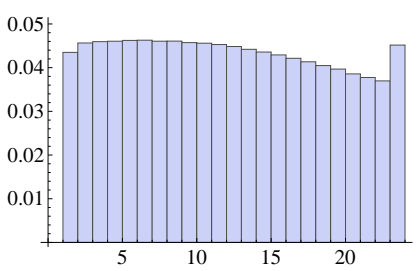

\%starve: $4.52 \%$

MDSD: 11.13 days

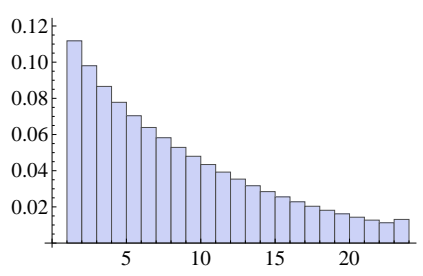

\%starve: $1.31 \%$

MDSD: 7.603 days

Figure 3: Frequency of duration of adult vector dispersals from 1 to 23 days for each vector/host combination (the last bar represents starvation/failure) and $t_{b}=2$ vs. 10 , along with failure proportion (\%starve) and mean duration of successful dispersals (MDSD)

1 limit vectors' ability to disperse far enough to switch, the fact that the overwhelming majority of bloodmeals involve a different host than the previous bloodmeal helps explain the spread of parasites such as T. cruzi within a host population.

\section{${ }_{4} 4$ Discussion}

5 The preliminary results reported in this manuscript offer both quantitative and qualitative de6 scriptions of vector-host interactions that are impractical to obtain via field studies yet critical 7 to understanding the sylvatic transmission of parasites like T. cruzi within and between reservoir 8 cycles. To our knowledge, no other studies of any kind have tried to estimate actual sylvatic host- 
vector contact rates in the field. In addition to offering baseline estimates of contact rates, the simulations made using the agent-based model described herein confirm host irritability as a main limiting factor (in conjunction with host density) for vector population density, which in turn affects the contact rates per vector or per host. The relative scarcity of hosts like raccoons constrains vector growth even for high bite tolerance, while the relative abundance of hosts like woodrats allows host irritability to play a crucial role in the growth or decline of vector densities, as well as more specifically the success or failure (and duration) of vector dispersal, which is the root of the host-switching that spreads the parasite.

Simulations of the transmission cycle involving raccoons and T. sanguisuga showed that starvation/host unavailability would significantly reduce juvenile and adult vector densities well below the densities reported in association with woodrats, regardless of host irritability threshold; this suggests that the actual vector densities in raccoon dens are much lower, although further work is needed to estimate true vector densities when other hosts such as opossums and skunks which sometimes use the same dens as raccoons (not necessarily at the same time) are included.

The decline in vector densities when woodrats' bite tolerance is limited to 2 per night, combined with their growth when that tolerance is raised to 10, suggests that woodrats' actual tolerance is somewhere in between the two values, and further fine tuning can produce an estimate of the irritability threshold consistent with observed densities. The difference in densities and biting rates for T. sanguisuga and T. gerstaeckeri in woodrat nests is also worth noting, with values for the latter species (TG) consistently rising over time in situations where those for the former species (TS) continue a decline despite being more common in nests.

Quantitatively, then, the per-host contact rates approach the host irritability threshold, except when both that threshold and host density are high; likewise, when vector densities reach sustainable levels, the per-vector contact rates are on average just high enough to avoid starvation, but not 
1 high enough to avoid the need for dispersal. The exception was the scenario with high host density

2 (woodrats) and high bite tolerance $\left(t_{b}=10\right)$, for which the TS mean biting rates hovered around

3 the frequency $(14 \text { days })^{-1}$ matching the dispersal threshold while TG mean biting rates were

4 approximately $(22 \text { days })^{-1}$ over the 100 -day simulations.

$5 \quad$ Further work using this model will examine in detail the rates at which individual vectors

6 change hosts, as well as the rates of host type switching, the mechanism for spreading parasites 7 across cycles.

\section{Acknowledgments}

KY was supported by NSF grant DMS-0946431; AM and CK acknowledge the support of NSF grant DMS-1020880. The authors also acknowledge the work of their student coauthors in developing [38], a preliminary version of the present study.

\section{References}

[1] D. L. DeAngelis and L. J. Gross, Individual-based models and approaches in ecology: populations, communities and ecosystems, Taylor and Francis, 1992.

[2] Constantin Barbu, Eric Dumonteil, Sébastien Gourbière, Characterization of the dispersal of non-domiciliated Triatoma dimidiata through the selection of spatially explicit models, PLoS Negl Trop Dis 4(8): e777, 2010. doi:10.1371/journal.pntd.0000777

[3] E.C. Borges, J.P. Dujardin, C.J. Schofield, A.J. Romanha, L. Diotaiuti, Dynamics between sylvatic, peridomestic and domestic populations of Triatoma brasiliensis (Hemiptera: Reduviidae) in Ceara state, northeastern Brazil, Acta Tropica 93: 119-126, 2005.

[4] T.W. Box, Density of plains wood rat dens on four plant communities in South Texas, Ecol. 40(4): 715-716, 1959. 
1 [5] J.K. Braun, M.A. Mares, Neotoma micropus, Mammalian Species 330: 1-9, 1989.

[6] J.E. Burkholder, T.C. Allison, and V.P. Kelly, Trypanosoma cruzi (Chagas) in invertebrate, reservoir, and human hosts of the Lower Rio Grande Valley of Texas, J. Parasitology 66(2): 305-311, 1980.

[7] M. Canals, R. Solís, C. Tapia, M. Ehrenfeld, P.E. Cattan, Comparison of some behavioral and physiological feeding parameters of Triatoma infestans Klug, 1834 and Mepraia spinolai Porter, 1934, vectors of Chagas disease in Chile, Memorias do Instituto Oswaldo Cruz 94(5): 687-692, Sep./Oct. 1999.

[8] Mónica B. Castañera, Juan P. Aparicio, Ricardo E. Gürtler, A stage-structured stochastic model of the population dynamics of Triatoma infestans, the main vector of Chagas disease, Ecological Modelling 162: 33-53, 2003.

[9] S.S. Catalá, The biting rate of Triatoma infestans in Argentina, Med. Vet. Entomol. 5: 325333, 1991.

[10] L.A. Ceballos, G.M. Vazquez-Prokopec, M.C. Cecere, P.L. Marcet, R.E. Gürtler, Feeding rates, nutritional status and night dispersal potential of peridomestic populations of Triatoma infestans in rural northwestern Argentina, Acta Tropica 95(2): 149-159, 2005.

[11] S.A. Conditt, D.O. Ribble, Social organization of Neotoma micropus, the southern plains woodrat, American Midland Naturalist 137(2): 290-297, 1997.

[12] R.B. Eads, H.A. Trevino, and E.G. Campos. Triatoma (Hemiptera: Reduviidae) infected with Trypanosoma cruzi in south Texas wood rat dens, The Southwestern Naturalist 8(1): 38-42, 1963. 
1 [13] Hugo Devillers, Jean Raymond Lobry, Frédéric Menu, An agent-based model for predicting 2 the prevalence of Trypanosoma cruzi I and II in their host and vector populations, Journal of Theoretical Biology 255: 307-315, 2008.

[14] Holly Gaff, Preliminary analysis of an agent-based model for a tick-borne disease, Mathematical Biosciences and Engineering 8(2): 463-473, April 2011. doi:10.3934/mbe.2011.8.463

[15] Cleber Galvão, Dayse da Silva Rocha, José Jurberg, Rodolfo Carcavallo, Início da Atividade de Vôo em Triatoma infestans (Klug, 1834) e T. melanosoma Martínez, Olmedo \& Carcavallo, 1987 (Hemiptera, Reduviidae), Memorias do Instituto Oswaldo Cruz 96(1): 137-140, January 2001. Online at http://memorias-old.ioc.fiocruz.br/961/4050.pdf.

[16] Viviane Galvão, José Garcia Vivas Miranda and Ricardo Ribeiro-dos-Santos, Development of a two-dimensional agent-based model for chronic chagasic cardiomyopathy after stem cell transplantation, Bioinformatics 24(18): 2051-2056, 2008. doi:10.1093/bioinformatics/btn362

[17] V. Grimm, U. Berger, F. Bastiansen, S. Eliassen, V. Ginot, J. Giske, J. Goss-Custard, T. Grand, S. K. Heinz, G. Huse, A. Huth, J. U. Jepsen, C. Jørgensen, W. M. Mooij, B. Müller, G. Peer, C. Piou, S. F. Railsback, A. M. Robbins, M. M. Robbins, E. Rossmanith, N. Rüger, E. Strand, S. Souissi, R. A. Stillman, R. Vabø, U. Visser and D. L. DeAngelis, A standard protocol for describing individual-based and agent-based models, Ecological Modelling 198: 115-126, 2006.

[18] V. Grimm and S. F. Railsbeck, Individual-based modeling and ecology, Princeton University Press, 2005.

[19] Volker Grimm, Uta Berger, Donald L. DeAngelis, J. Gary Polhill, Jarl Giske, Steven F. Railsback, The ODD protocol: a review and first update, Ecological Modelling 221: 2760-2768, 2010. 
1 [20] Pablo G. Guerenstein and Claudio R. Lazzari, Host seeking: how triatomines acquire and $2 \quad$ make use of information to find blood, Acta Tropica 110: 148-158, 2009.

3 [21] S.A. Kjos, K.F. Snowden, J.K. Olson, Biogeography and Trypanosoma cruzi infection preva-

4

5 lence of Chagas disease vectors in Texas, USA, Vector-Borne and Zoonotic Diseases 9(1): 41-50, 2009.

[22] C.M. Kribs-Zaleta, To switch or taper off: the dynamics of saturation, Math. Biosci. 192(2): $137-152,2004$.

[23] C.M. Kribs-Zaleta, Vector consumption and contact process saturation in sylvatic transmission of T. cruzi, Mathematical Population Studies 13(3): 135-152, 2006.

[24] C.M. Kribs-Zaleta, Sharpness of saturation in harvesting and predation, Math. Biosci. Eng. 6(4): 719-742, 2009.

[25] C.M. Kribs-Zaleta, Estimating contact process saturation in sylvatic transmission of Trypanosoma cruzi in the U.S., PLoS Neglected Tropical Diseases 4(4): e656, 2010.

[26] C.M. Kribs-Zaleta, Alternative transmission modes for Trypanosoma cruzi, Math. Biosci. Eng. 7(3): 661-676, 2010.

[27] Claudio Ricardo Lazzari, Marcos Horácio Pereira, Marcelo Gustavo Lorenzo, Behavioural biology of Chagas disease vectors, Memorias do Instituto Oswaldo Cruz 108(Suppl. I): 34-47, 2013.

[28] M.J. Lehane, C.J. Schofield, Measurement of speed and duration of flight by triatomine bugs, Transactions of the Royal Society of Tropical Medicine and Hygiene 72: 438, 1978.

[29] A. López, L. Crocco, G. Morales, S. Catalá, Feeding frequency and nutritional status of peridomestic populations of Triatoma infestans from Argentina, Acta Tropica 73: 275-281, 1999. 
1 [30] José Alejandro Martínez-Ibarra, Ricardo Alejandre-Aguilar, Edgar Paredes-González, Miguel

2 Alejandro Martínez-Silva, Miriam Solorio-Cibrián, Benjamín Nogueda-Torres, Francisco Trujillo-Contreras, Mónica Novelo-López, Biology of three species of North American Triatominae (Hemiptera: Reduviidae: Triatominae) fed on rabbits, Memorias do Instituto Oswaldo Cruz 102: 925-930, 2007.

[31] Robert Merkelz and Sara F. Kerr, Demographics, den use, movements, and absence of Leishmania mexicana in southern plains woodrats (Neotoma micropus), The Southwestern Naturalist, 47(1): 70-77, 2002.

[32] F. Noireau and J.P. Dujardin, Biology of Triatominae, in J. Telleria and M. Tibayrenc (eds.), American Trypanosomiasis Chagas Disease: one hundred years of research, Elsevier, Boston, 2010, pp. 149-168.

[33] P. Pelosse and C.M. Kribs-Zaleta, The role of the ratio of vector and host densities in the evolution of transmission modes in vector-borne diseases. The example of sylvatic Trypanosoma cruzi, Journal of Theoretical Biology 312: 133-142, 07 November 2012. doi:10.1016/j.jtbi.2012.07.028.

[34] W.F. Pippin, The biology and vector capability of Triatoma sanguisuga texana Usinger and Triatoma gerstaeckeri compared with Rhodnius prolixus, Journal of Medical Entomology 7(1): $30-45,1970$.

[35] Alan R. Rabinowitz and Michael R. Pelton, Day-bed use by raccoons, Journal of Mammalogy 67(4): 766-769, 1986.

[36] M.J. Ramirez-Sierra, M. Herrera-Aguilar, S. Gourbière, E. Dumonteil, Patterns of house infestation dynamics by non-domiciliated Triatoma dimidiata reveal a spatial gradient of infestation in rural villages and potential insect manipulation by Trypanosoma cruzi, Tropical Medicine and International Health 15: 77-86, 2010. 
1 [37] G.G. Raun, A population of woodrats (Neotoma micropus) in southern Texas, Texas Mem. $2 \quad$ Mus. Bull., 1966.

[38] Catherine Rogers, Kim Ngan Tran Nguy, Onyekachi A. Amajor, Thomas Seaquist, Britnee Crawford, Anuj Mubayi, Christopher Kribs-Zaleta, and Oladipo Oyediran, Daily behavior of Trypanosoma cruzi hosts and vectors in Texas: an agent-based modeling approach in NetLogo, Mathematics Department Technical Report 2010-18, University of Texas at Arlington, 2010. Online at http://www.uta.edu/math/preprint/rep2010_18.pdf

[39] C.J. Schofield, The role of blood intake in density regulation of populations of Triatoma infestans (Klug) (Hemiptera: Reduviidae), Bull. Ent. Res. 72: 617-629, 1982.

[40] C.J. Schofield, N.G. Williams and T.F. de C. Marshall, Density-dependent perception of triatomine bug bites, Annals of ropical Medicine and Parasitology 80(3): 351-358, 1986.

[41] C.J. Schofield, M.J. Lehane, P. McEwen, S.S. Catala, D.E. Gorla, Dispersive flight by Triatoma sordida, Transactions of the Royal Society of Tropical Medicine and Hygiene 85: 676-678, 1991.

[42] C.J. Schofield, M.J. Lehane, P. McEwen, S.S. Catala, D.E. Gorla, Dispersive flight by Triatoma infestans under natural climatic conditions in Argentina, Medical and Veterinary Entomology 6: 51-56, 1992 .

[43] H.W. Shirer and H.S. Fitch, Comparison from radio tracking of movements and denning habits of the raccoon, striped skunk, and opossum in northeastern Kansas, J. Mammal. 51: 491-503, 1970.

[44] R. Slimi, S. El Yacoubi, E. Dumonteil, S. Gourbière, A cellular automata model for Chagas disease, Applied Mathematical Modelling 33: 1072-1085, 2009. 
1 [45] Kathleen M. Thies, Monte L. Thies, and William Caire, House construction by the southern 2 plains woodrat (Neotoma micropus) in southwestern Oklahoma, The Southwestern Naturalist

3

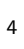

5

\section{Appendix. Parameter estimation}

\section{Host densities and homes}

[46] Uri Wilensky, NetLogo, Center for Connected Learning and Computer-Based Modeling, Northwestern University, Evanston, IL, 1999. http://ccl.northwestern.edu/netlogo.

As noted in the main text, cell diameters were set at $10.4 \mathrm{~m}$ in the model since this reflects [38] the approximate minimum distance between woodrat nests in one study [31], the mean distance between nests in another study [45], and the mean distance traveled daily by a woodrat in a third [37]. With a $75 \times 75$ grid, the resulting area is about 60 ha or 150 acres. Population densities can be multiplied by the proportion of this area which contains suitable habitat, in order to figure absolute population sizes.

Woodrat population density estimates vary by more than an order of magnitude, cf. reviews in $[25,38]$. (Some of the variation in published estimates may be explained by seasonal fluctuations.) Here we use 9.3 woodrats/acre (23 woodrats/ha), which is both the mean found across all studies considered in [25] and the density found in [37], although the cross-study mean found in [38] is lower, 5.79 woodrats/acre. Likewise the studies reviewed in [25] found raccoon densities varying from 0.002 to 0.083 raccoons/acre in sylvatic sites and up to 0.5 raccoons per acre in peridomestic settings and national parks in the U.S.; we here use the average of 0.08 raccoons/acre ( 0.2 raccoons/ha) in sylvatic sites given in [25].

The southern plains woodrat, Neotoma micropus, lives primarily in shortgrass landscapes dominated by prickly pear cactus, where they make their nests (and which serves as primary food 
1 source) $[4,5,11,37]$. These woodrats live generally alone in single nests, although males may range 2 over multiple nests, some of them shared with females [5, 31, 37]. An extensive 20-month study by

3 Merkelz and Kerr found some nests used by a succession of 5 or more different rats during the study

4 [31]. They also found a roughly 1:1 sex ratio, with $24 \%$ of recaptured females and $69 \%$ of recaptured

5 males found at multiple nests. Overall they tagged 180 rats at 86 nests, for a nests/woodrat ratio 6 of 2.1 .

7 The probability of a woodrat changing nests from one day to the next (for reasons other than 8 host irritability) was estimated at $1 / 4$ by supposing that half of woodrats are female and do not 9 normally change nests, while the other half are male and change nests on average every 2 days. We

found no empirical estimates in the literature for this quantity. The only major study of woodrat nest use (Merkelz and Kerr [31]) made 239 captures at 86 nests over 2292 trap-nights during 20 months, so each nest was checked an average of $2292 / 86=26.65$ times, for an average frequency of 1.33 times per month, not often enough to derive estimates for daily change rates.

Shirer and Fitch [43] studied the movements of four adult raccoons, observed respectively in 4 , 7, 12, and 14 different locations, for an average of 9.25 dens per raccoon. They also studied four juvenile raccoons but did not disentangle the number of locations recorded for each. A literature review did not find any data on the proportion of raccoon dens (or opossum or skunk, which often share dens, not necessarily simultaneously) infested with triatomines, so we use the proportion for woodrat nests as a proxy.

\section{Vector density}

Eads et al. [12] dismantled 80 woodrat nests and found 390 Triatoma in 58 of 80 nests (72.5\%): by life stage, 372 juveniles and 18 adults total, which gives a paltry 6.4 (mean) juveniles and 0.31 adults per infested nest, and 4.875 total vectors per overall nest; by species, 226 T. sanguisuga, 133 T. gerstaeckeri, 31 Triatoma neotomae by species (T. neotomae specialize in woodrats but 
are rare in Texas, more common in Mexico), which gives T. sanguisuga a 1.7:1 advantage over $T$. gerstaeckeri $(63.0 \% / 37.0 \%$ excluding T. neotomae) in nests. On first glance the small numbers might seem to contradict Burkholder et al.'s [6] estimated density (it would take 26.25 nests per acre to replicate the observed 128 bugs/acre), but in fact Eads et al. observe that what it really means is that adults are frequently out foraging and not in the nest all the time. So we should expect to see lots of dispersal in our simulations.

Pippin [34] searched 142 nests and found 229 bugs in 85 nests (68.5\%): by life stage, 213 nymphs and 16 adults total, or 2.5 juveniles and 0.19 adults per infested nest (range 1-22 total), 1.612 total vectors per overall nest; by species, $123+9=132$ T. sanguisuga (nymphs+adults), 90+7=97 $T$. gerstaeckeri, for a 1.36:1 ratio T. sanguisuga:T. gerstaeckeri (57.6\%/42.4\%). The study includes the following passage supporting the notion of widespread adult dispersal:

"The bugs were rarely found in the rats' nests. The preferred habitat appeared to be in cracks and crevices in tunnels and runways leading to the nest or in debris covering the nest. Nymphs were occasionally found under logs and debris as far as $90 \mathrm{~m}$ from the nest of any known host. The condition of most of the bugs indicated they had not fed for some time. This was true even when the bugs were within $60 \mathrm{~cm}$ of an occupied nest."

We take the averages from these two papers of $70.5 \%$ for nest infestation proportion and $60 \% / 40 \%$ T. sanguisuga-T. gerstaeckeri ratio. Our estimates of 128 vectors/acre, 9.3 woodrats/acre, 2.1 nests/woodrat and $70.5 \%$ nest infestation, however, produce an expected average 9.3 vectors per infested nest, compared to Eads et al.'s 6.71 and Pippin's 2.69. Since the latter two studies concentrated around nests, we interpret the difference to indicate adults dispersed away from nests, and use the higher figure to seed the model (all vectors begin in nests but adults may eventually disperse to match observed data). Thus of 9.3 vectors per average infested nest, we suppose a mean (of 6.4 and 2.5) 4.45 juveniles and $9.3-4.45=4.85$ adults, making juveniles represent $47.85 \%$ of 
1 the vector population.

\section{Host irritability, vector feeding and dispersal}

3 In studying host irritability, [8] estimated bites of the first through third instars to contribute only $41 / 10$ as much to host irritability as an adult vector's bite, and bites of fourth and fifth instars 5 to contribute 0.8 times as much. (In comparison, Pippin found [34, Table 8] that the volume of

blood taken per bloodmeal increased by about $1 / 2$ an order of magnitude per instar stage for $T$. sanguisuga, and half as much for T. gerstaeckeri.) They also estimated host irritability at between 2 and 10 bites per day per host (described as vector density but given in units of per day). Their results indicated that differentiating between nymphs' and adults' contributions to host irritability was crucial to reproducing observed patterns in experimental data (although precise differences between nymph stages were not), and that the host irritability threshold does play an important role in vector density.

Here, we use Pippin's [34, Table 5] data on mean duration of each instar stage for T. sanguisuga under varying $\left(18-30^{\circ} \mathrm{C}\right)$ and constant $\left(27^{\circ} \mathrm{C}\right)$ temperatures, and $T$. gerstaeckeri under varying temperatures, to estimate $45 \%$ of juveniles in the first three instars and $55 \%$ in the last two, together with relative irritation factors of 0.1 and 0.8 from [8], to estimate an overall irritation factor of 0.485 for juveniles relative to adult bites, or 2.06 juvenile bites irritation-equivalent to 1 adult bite. (Data for T. gerstaeckeri under constant temperature exhibited a markedly different pattern, with only $24.3 \%$ of a juvenile's life spent in the first three instars.) In comparison, a similar stagewise average of bloodmeal size weighted by mean stage duration using Pippin's data produces an equivalence factor of 1.24 or 1.56 for T. sanguisuga and 1.77 or 2.48 for T. gerstaeckeri juvenile bloodmeals to 1 adult bloodmeal.

Although we found no direct studies of preferred feeding frequency for T. sanguisuga or T. gerstaeckeri, a 7-day interval is consistent with results from the literature for other triatomine 
1 species. Borges et al. reported a feeding frequency of once per week for Triatoma brasiliensis [3]. 2 Other studies reported mean times between bloodmeals ranging from 6.24 to 10.74 days for some South American triatomines [7], 4 to 9 days for some domestic South American triatomines [9], or

1.7 to 3.4 days for peridomestic populations of Triatoma infestans in Argentina during spring and summer [29]. Ceballos et al. [10] wrote of Triatoma infestans, "Mean daily feeding rates decreased significantly from $34 \%$ in spring to $23 \%$ in summer to $14 \%$ and $18 \%$ in fall and winter, respectively; mean feeding intervals were 2.9, 4.3, 7.0 and 5.6 days, respectively."

Pippin [34] captured 698 T. gerstaeckeri using outdoor light traps at night and found that only 2 had recently fed. He wrote that their general appearance was similar to bugs which had not fed for 14-21 days. This is consistent with Galvão et al.'s study of Triatoma infestans and the closely related Triatoma melanosoma [15], which found that the peak vector dispersal time was 14 days after feeding. The ten unfed females which Pippin then tested for longevity survived from 4 to 22 days with a mean of 13 . If we suppose that bugs within less than 4 days of starvation were not attracted to the traps, this allows up to at least 22 days survival post-dispersal before starvation. (Note that the data in Pippin's Table 12 for survival on one feeding, with means well over 100 days for adults of both species, were collected under field conditions during the winter, beginning on November 1, 1966; the discrepancy with the lower values here is consistent with other results from Pippin which suggest that the bugs go dormant in the winter. Whether or not it is true diapause remains to be studied.) From all this we assume that dispersal initiates 14 days after feeding, and starvation occurs 36 days after feeding.

Pippin studied starvation longevity of adults in the lab, and of adults and instars of all stages in the field over the winter (beginning Nov. 1); for T. sanguisuga there was a consistent factor of 4.5 between lab longevity and outdoor winter longevity for both sexes of adult, so applying the same factor to the winter survival of nymphs, the average extrapolated starvation longevity for juveniles is 31.5 days, consistent with the estimate above for adults. 


\begin{tabular}{|l|c|c|}
\hline Parameter & $\begin{array}{c}\text { Base rate } \\
\text { (from lit.) }\end{array}$ & $\begin{array}{c}\text { Probability } \\
\text { (1 day) }\end{array}$ \\
\hline Raccoon birth & $0.9 / \mathrm{yr}$ & 0.00246 \\
Woodrat birth & $1.8 / \mathrm{yr}$ & 0.00492 \\
Raccoon death & $0.4 / \mathrm{yr}$ & 0.00109 \\
Woodrat death & $1 / \mathrm{yr}$ & 0.00273 \\
T.s. birth & $54 / \mathrm{yr}$ & 0.137 \\
T.g. birth & $160 / \mathrm{yr}$ & 0.355 \\
T.s. juv. death & $1.5 /(2.25 \mathrm{yr})$ & 0.00182 \\
T.g. juv. death & $1.5 /(361.9 \mathrm{dy})$ & 0.00414 \\
T.s. maturation & $1 /(2.25 \mathrm{yr})$ & 0.00122 \\
T.g. maturation & $1 /(361.9 \mathrm{dy})$ & 0.00276 \\
T.s. adult death & $1 /(525 \mathrm{dy})$ & 0.00190 \\
T.g. adult death & $1 /(285.2 \mathrm{dy})$ & 0.00350 \\
\hline
\end{tabular}

Table 2: Conversion of baseline event rates from literature into probabilities of the events occurring in a single day (T.s.=T. sanguisuga, T.g.=T. gerstaeckeri)

A literature review found no studies with information on dispersal distances for T. sanguisuga

or T. gerstaeckeri. Schofield, Lehane and colleagues conducted several studies on dispersal of South

American species including T. infestans and T. sordida and found, in general, that of the bugs they released to disperse, about half went nowhere (remaining $5 \mathrm{~m}$ or less from the release spot) and the other half flew $50 \mathrm{~m}$ or more (most of them flew $100 \mathrm{~m}$ or more and were lost to the researchers); in one case a vector was found to have traveled $1350 \mathrm{~m}[28,41,42]$. Aware of the possible effect of dispersal distance, we take a mean of $1000 \mathrm{~m}$, but note that preliminary simulations using much lower values resulted in near-universal failure to find new hosts.

\section{Rates converted to probabilities}

The vector reproduction rates (per capita per year) were calculated by taking the expressions given in $[25$, Supp.] and removing the $60 \%$ survival-to-maturity probability. Likewise vector longevities in the same source were broken down into time-to-maturation and adult lifespan. For each vector species, the $60 \%$ survival-to-maturity proportion was then converted into a juvenile mortality rate $\mu_{j}$ by setting $\frac{\mu_{j}}{\mu_{j}+m}=0.6$ (where $m$ is the maturation rate), whence $\mu_{j}=1.5 m$. Host reproduction and mortality rates were taken directly from [25]. All base rates are given in Table 2. 
To convert these rates into daily probabilities, first the units were converted to days ${ }^{-1}$ (by dividing per-year rates by 365.24). Then for each rate, say $\gamma$, the probability of a given transition/event occurring or not occurring was determined using a simple differential equation $\frac{d x}{d t}=-\gamma x$, whence the proportion of the population which has not made the transition after $T$ units of time is $x(T) / x(0)=e^{-\gamma T}$, and the proportion that has made the transition (e.g., reproducing, maturing, dying) is then $1-e^{-\gamma T}$. (Survival is thus defined in terms of not dying.) These proportions were used for probabilities, with $\gamma$ in days $^{-1}$ and $T=1$ day. This derivation corresponds to the probabilities in a Poisson waiting process with parameter $\gamma$.

Finally, since starvation and natural mortality [due to causes other than starvation] are modeled separately in the ABM, we adjusted the vector survival probabilities by increasing them to offset the observed starvation rates, which were remarkably consistent across simulations. T. sanguisuga's daily per capita starvation rate in the model was observed at 0.00189 , accounting for nearly all adult deaths (total mortality estimated at 0.00190 , cf. Table 2), so the adult daily survival probability [due to other causes] rose to 0.99999 . 
Mean adult TS population, $\mathrm{tb}=2$

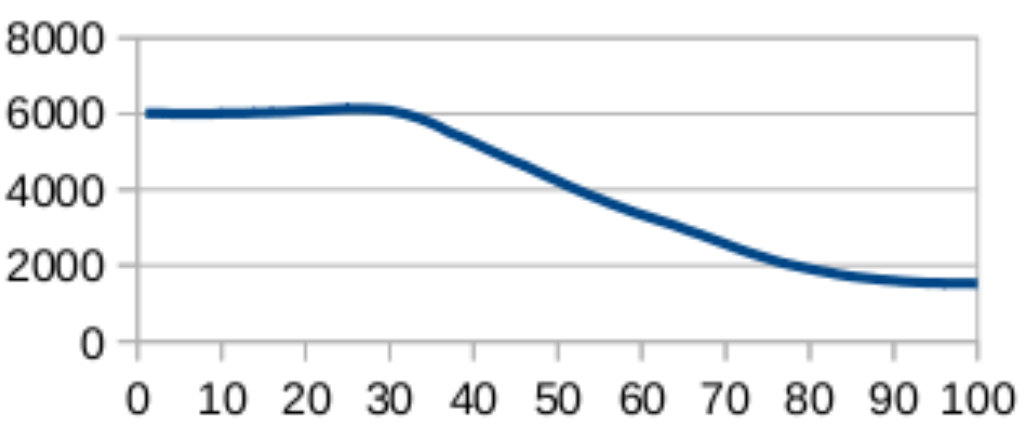

Mean adult TS population, $\mathrm{tb}=10$

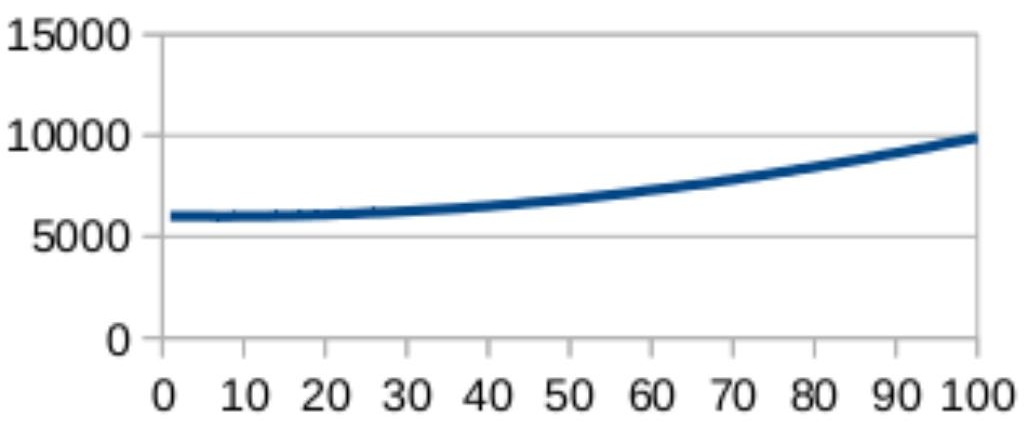

Mean adult TG population, $\mathrm{tb}=2$

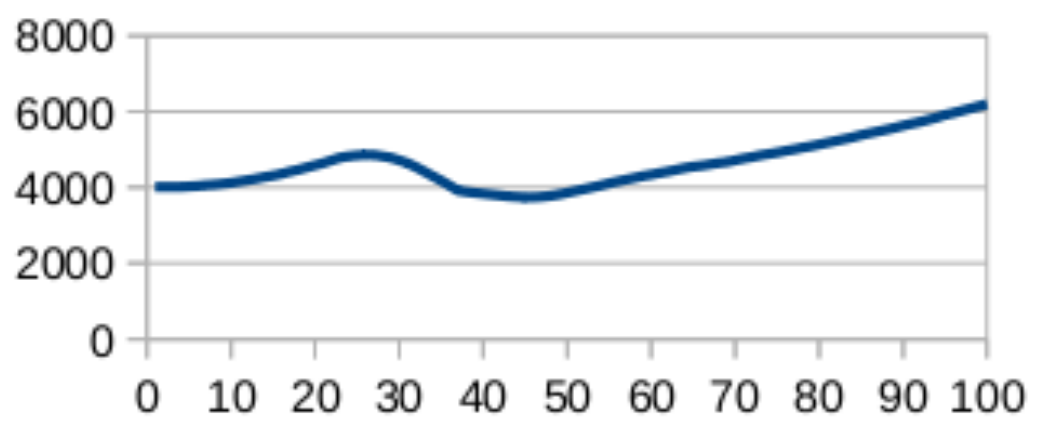

Mean adult TG population, $\mathrm{tb}=10$

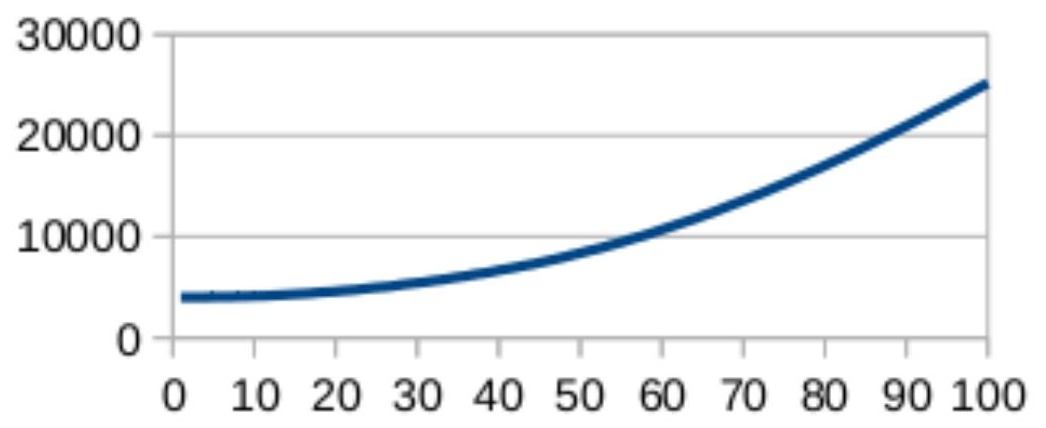

